\title{
ABOUT THE SUSPECTED VERY YOUNG PN IRAS 17516-2525
}

\author{
HANS ULRICH KÄUFL \\ European Southern Observatory, D-8046 Garching, Germany \\ and \\ LETIZIA STANGHELLINI \\ Osservatorio Astronomico di Bologna, I-40126 Bologna, Italy
}

Infrared photometry suggests that IRAS $17516-2525$ is in transition from the AGB to a young PN (Van der Veen et al. 1989a,b, Manchado et al. 1989). IR spectra (van der Veen et al. 1989b, Käufl et al. 1992) revealed that the object has an ionized core. Optical observations show a weak $\mathrm{H}_{\alpha}$ emitting unresolved object (Käufl et al. 1992$)$ at the coordinates $\left(\alpha_{1950}: 17^{h} 51^{m} 37.8^{s}, \delta_{1950}:-25^{\circ} 25^{\prime} 58^{\prime \prime}\right)$ suggested by van der Veen et al. (1989a). The spectrum between 2.0 and $2.4 \mu m$ shows e.g. $\mathrm{Br}_{\gamma}$, $2.0875 \mu m$ (unidentified) or the $\mathrm{Na}_{I}$-doublet but no molecular Hydrogen (present in other compact $\mathrm{PNs}$ ). The ratio of $\mathrm{Pf}_{\beta}$ to $\mathrm{Br}_{\alpha}$ (hardly affected by extinction, insensitive to electron density and temperature) has been studied in IRAS 17516-2525 and Hen1044. For Hen1044 we find this ratio to be in accordance with theoretical calculations (Hummer and Storey, 1987). For IRAS 17516-2525, however, $\operatorname{Pf}_{\beta}$ is approximately twice as strong. Hence presumably Hen1044 is optically thin whereas IRAS $17516-2525$ is optically thick at $\lambda \approx 4-5 \mu m$. Line fluxes appear to be constant over a time scale of 4 years. If the visual extinction $A_{V}$ in the object is $\geq 2^{\text {mag }}$ than the infrared luminosity equals the total luminosity (i.e. $\left.L=1400 L_{\odot} *\left(\frac{\text { distance }}{k p c}\right)^{2}\right)$. A main-sequence object accidentally extincted by an intervening cloud can be excluded because the observed line-width of $\mathrm{Br}_{\alpha}\left(\approx 40 \frac{\mathrm{km}}{\mathrm{s}}\right.$, Van der Veen et al. 1989a) is too small as compared to that of O-stars $\left(\approx 1000 \frac{\mathrm{km}}{\mathrm{s}}, \mathrm{Käufl}, 1992\right)$. Assuming an expansion-velocity of $\approx 20 \frac{\mathrm{km}}{\mathrm{s}}$ the stellar appearance constrains dynamic age and size $\left(\right.$ age $\leq 360 y * \frac{\text { distance }}{k p c}$, size $\left.\leq 1500 A U * \frac{\text { distance }}{k p c}\right)$. A pre-main sequence object can also be excluded. The max. luminosity $\left(L_{P M S} \leq 5000 L_{\odot}\right)$ then requires the object to be closer than $2 \mathrm{kpc}$ where it should be resolvable. In conclusion the observational evidence strongly suggests that IRAS 17516-2525 represents the searched 'missing link' between AGB stars and young compact Planetary Nebulae.

\section{References}

Hummer, D.G., and Storey,-P.J: 1987, MNRAS 224, 801

Käufl, H.U. et al.: 1992, in Mass Loss on the AGB and Beyond, ed(s)., H.E. Schwarz, in press, Käufl, H.U.: 1992, Astron. Astrophys. in press,

Manchado, A. et al.: 1989, Astron. Astrophys. 214, 139

Van der Veen, W.E.C.J. et al.: 1989a, Astron. Astrophys. 216, L1

Van der Veen, W.E.C.J. et al.: 1989b, Astron. Astrophys. 226, 108 